\title{
THE MORPHOLOGY, PATHOGENESIS OF \\ TERMINOLOGY AND ARTERIAL PLAQUES
}

\author{
C.'J. Schwartz,'M.D. and J. R. A. Mitchell, M.B., B.Sc., M.R.C.P. \\ Department of the Regius Professor of Medicine, Radcliffe Infirmary, Oxford
}

\begin{abstract}
'. . a scientific survey which is to throw light on the problem of arteriosclerosis in all its various aspects must be based upon a clear delimitation of that term'. -Aschoff (1933).

THE lack of precision of terms such as arteriosclerosis, atheroma and atherosclerosis was emphasized by Rabson (1954), who showed that they have different meanings for different pathologists, and they are used to describe widely different types of lesions, including many bizarre varieties of experimentally induced arterial disease.
\end{abstract}

Lobstein (1833) first coined the word arteriosclerosis, which he applied as a generic term to all forms of arterial hardening. From its inception, therefore, the term arteriosclerosis lacked precision and this difficulty was recognized by Klotz (1906), who, in suggesting that it be retained in a generic sense, emphasized the need for recognition of the different varieties of disease which were to be included under this general heading. Mönckeberg (1903) described one form of arterial hardening, namely, medial calcification, and stressed that this change was a pathological entity unrelated to the other types of arteriosclerosis. If the term arteriosclerosis is used in the sense in which it was introduced, to cover all types of arterial hardening, it serves no useful purpose; if used in any other context it serves only to confuse an already complex problem.

Of all the terms used to describe the various plaques occurring in the large arteries of man, atherosclerosis and atheroma are the least welldefined and most frequently misused. The word atherosclerosis was introduced by Felix Marchand (I904) to include the various arterial lesions considered as ' arteriosclerosis' and to emphasize the presence of lipid material in certain of the lesions. Atherosclerosis is therefore nothing more than a complex alternative for arteriosclerosis and suffers in consequence from the same lack of specificity.

According to Paré (1575), the term atheroma was used in the ancient Greek literature to describe any cystic space or sac containing a gruel-like material. It was first applied to arterial pathology by von Haller (1755) to describe a particular type of arterial plaque which on sectioning exuded a yellow pultaceous material. It is in this sense that atheroma has been recently defined by WHO $\left(195^{8}\right)$ as a ' ... plaque in which fatty softening is predominant'. All would be well if workers were to limit its use to a particular type of plaque, but atheroma, atherosclerosis and arteriosclerosis are currently used as synonyms for a wide variety of arterial lesions. These terms, therefore, lack a clear and reproducible meaning.

We considered, therefore, that an attempt should be made to describe the range of macroscopic plaques which can be recognized on the intimal surface of the major arteries (aorta, carotid, iliac) and define the histological characteristics of these plaques. Some observations resulting from such a study are presented.

\section{Macroscopic Types of Arterial Plaque}

Four types of lesion are recognizable on the luminal surface of the major arteries:

(i) Flat Sudanophilic Plaques (Fatty Streaks). In the unstained vessel these are seen as white or yellow areas. They show considerable variation in size and shape, from small spots no larger than a pin head to larger areas covering many square centimetres (Fig. I). These flat lesions stain intensely with the fat-stain Sudan-IV and are sharply demarcated from the surrounding intima. When an artery is sectioned transversely through such a plaque no other macroscopic feature is to be found, and in particular no areas of pultaceous material are seen in the depths.

(ii) Raised Sudanophilic Plaques are white or yellow in the unstained artery and with Sudan-IV staining they show a considerable variability in staining intensity. There is a wide variation in size and shape, but in general the smallest raised lesions are larger than the smallest flat plaques. In transverse section the deeper parts of such plaques commonly contain a white or yellow pultaceous material.

(iii) Raised White Plaques (Fibrous Plaques) have a firm non-sudanophilic surface. Transverse sections show that beneath the white surface layer there is almost invariably a mass of yellow pul- 


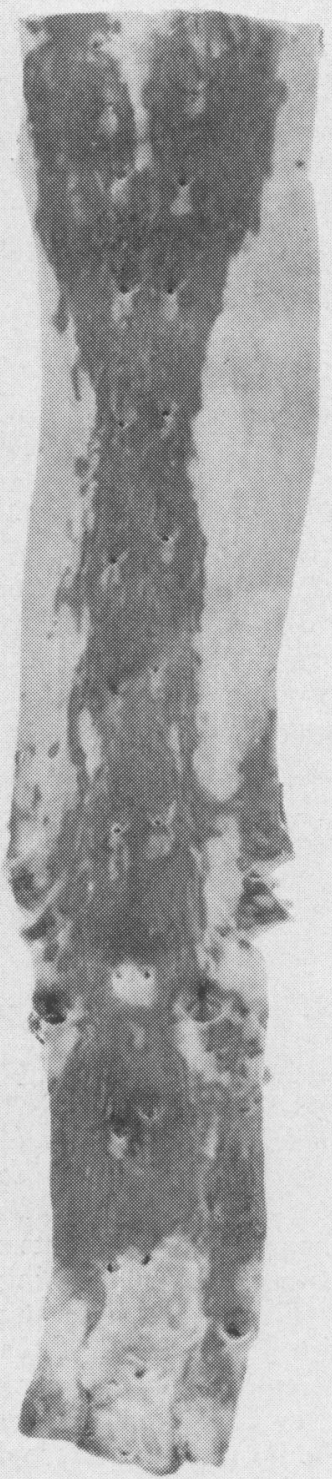

FIG. I.-Aorta from a male aged 30 , stained with SudanIV, showing fatty streaking. Note the fan-shaped distribution of sudanophilia in the thoracic segment, the sparing around the mouths of the intercostal arteries, and the localization of the fatty streaking along the midline posteriorly down to the level of the coeliac axis.

taceous material similar to that observed in the raised sudanophilic plaques.

(iv) Complicated Plaques are those showing calcification, surface ulceration, thrombosis or hæmorrhage. Calcification can be recognized as grey or white areas which are often umbilicated and, like ulceration, it has never been seen on the surface of a flat lesion. When ulceration is present on raised plaque it is commonly accompanied calcification.

\section{Microscopic Appearances of the Flat Sudanophilic Plaque}

These lesions affect the intima and there are $\frac{0}{10}$ characteristic changes in the other arterial coat. The intima shows a variable but slight thickening consisting of fat-laden macrophages in a fine meskfwork of fibrous and elastic tissue (Fig. Sudan-stained frozen sections show that these plaques contain many fat globules both in macros phages and extracellularly. There is usually a marked difference in the amount of lipid in the intimal and medial coats, the dividing line being the internal elastic lamina along which considerabpe fatty deposits may be found, even when the intimg? shows relatively little fat elsewhere, and this striking concentration of fat along the intimal side of the internal elastic lamina is a feature of th: type of lesion. The media may or may not sho $\overrightarrow{\mathrm{W}}$ fatty changes, and when medial fat is present occurs as fine globules, generally smaller than tho found in the intima and showing a tendency

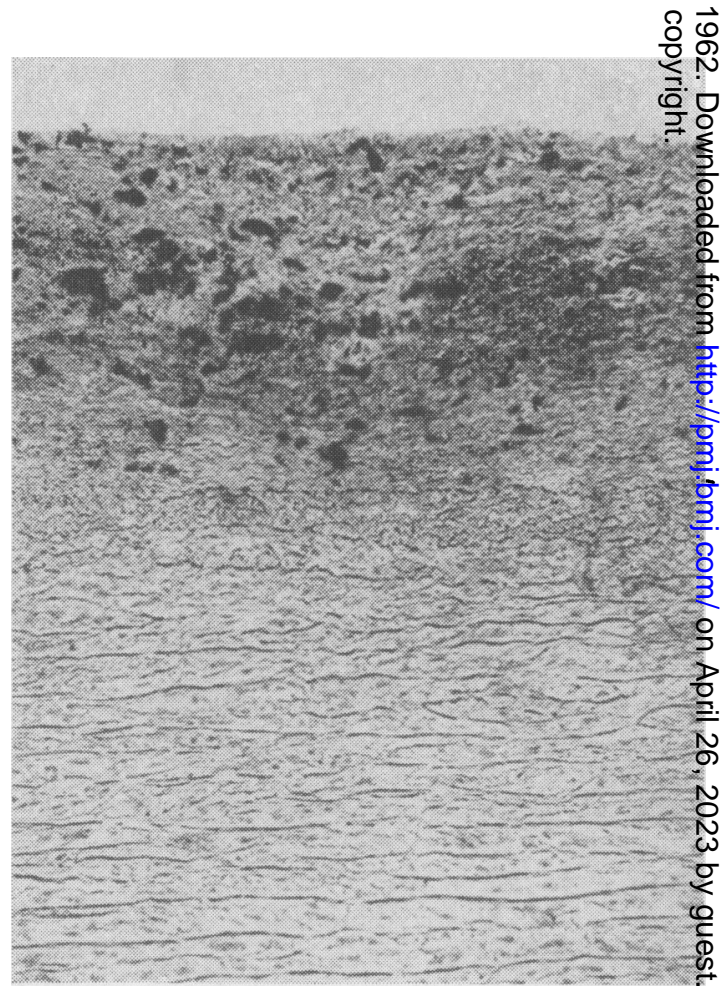

FIG. 2.-Frozen section through an aortic fatty streak of 66-year-old male. The lipid appears as blact globules in a fibro-elastic stroma. Note the norma media. Sudan-IV $\times$ Ioo. 
align along the medial elastic fibres. The medial fat appears to be largely extracellular. It should be emphasized that medial fat need not be associated with the presence of flat sudanophilic plaques in the intima, but can occur in otherwise normal arteries.

Within the intimal fatty streaks scattered small round cells morphologically similar to small lymphocytes, together with an occasional plasma cell and fibroblast, are sometimes seen. Other types of cells have not been encountered, while cellular infiltration of the media and adventitia does not occur. Occasionally the internal elastic lamina shows a hyaline thickening and fragmentation, but even in such instances the media is well preserved. Calcification of the media and internal elastic lamina has been seen in association with the flat sudanophilic plaque, particularly in the internal and common iliac arteries, but it has also been observed in these sites in the absence of intimal lesions, suggesting that it is not an integral component of this type of lesion.

On staining with azure-A and periodic acidSchiff (PAS) there is no constant tinctorial pattern, while material staining with phosphotungstic-acidhæmatoxylin (PTAH) - ' fibrin'-has never been found on the surface, within or beneath these plaques. These flat fatty plaques are devoid of histologically demonstrable vessels, while the vascularity of the medial and adventitial coats does not differ from that found in normal arteries.

\section{Microscopic Appearances of Raised Arterial Plaques}

Although three types of raised arterial plaque can be distinguished on the basis of their macroscopic appearance, no clear distinction can be made between them histologically, the three types having essentially similar characteristics.

(i) Fibrosis. Dense bundles of collagen are found in every elevated plaque, often forming thick layers on the luminal surface, and in the depths of the lesion, where they separate fatty masses from the media (Fig. 3). This fibrous tissue has a laminated structure and, although it contains some fibroblasts, is otherwise relatively acellular. Small lacunar spaces are commonly seen in this fibrous stroma and such areas do not stain with Sudan-IV in frozen sections. The dividing line between intima and media is often lost, the fibrous tissue of the plaque merging imperceptibly with the media (Fig. 3).

Orcein-elastic staining shows that in these areas of fibrosis, particularly in the depths of the plaque, there is a marked elastosis which again emphasizes the laminated structure of the lesion. Sudan-IV stained frozen sections show that the densely fibrotic areas are generally free from large accumu-

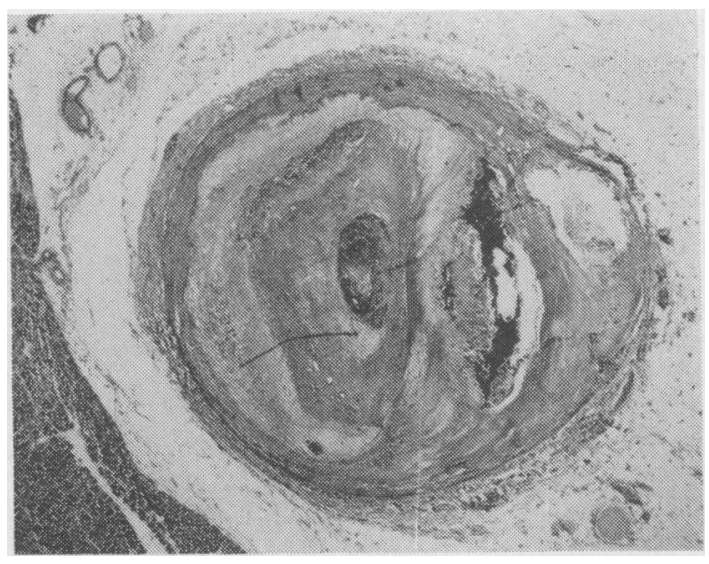

FIG. 3.-Human coronary artery, showing complete occlusion with thrombus, areas of revascularization, a large intramural hæmorrhage, cholesterol-clefted lipid deposits, extensive fibrosis and medial thinning. Scattered foci of adventitial lymphocytes are also present. Masson's trichrome $\times$ IO.

lations of fat, although there may be intra- and extracellular surface deposits, and it is these surface accumulations only which distinguish raised sudanophilic from raised white plaques.

Prominent metachromatic staining is very frequently found in the fibrous tissue of the raised plaques. The staining density is greatest in the depths of the plaque and around the lacunar spaces, while in general there is little surface metachromasia, except when organizing thrombus is present. The fibrous tissue is PTAH and generally PAS negative.

(ii) Elastosis. The laminated elastic tissue which occurs in areas of fibrosis has already been mentioned. In some sections the spacing and alignment of the laminæ suggests formation in successive layers in an episodic fashion, and in many instances such areas of elastosis closely resemble the changes which occur in organizing arterial thrombus.

(iii) Calcification can be seen in the plaque or in the artery wall. Within the plaque it may occur in the dense fibrous stroma or in the areas of cholesterol-clefted lipid deposits, and it is rarely found in areas of cellularity. Its extent and distribution within the lesions is very variable and it is commonly found microscopically when unsuspected on a macroscopic basis.

Medial calcification occasionally occurs by encroachment from the plaque itself, but is most commonly seen as an independent lesion. It has been found in many arteries, but is particularly common in the iliac arteries and the petrous and cavernous segments of the internal carotid artery, and is rarely seen in the coronary arteries. It shows no constant relationship to the presence or 
absence of a raised plaque, suggesting that it is not an integral part of these lesions, but simply reflects an association of two relatively common but unrelated processes.

Calcification of the internal elastic lamina is commonly found in the common and internal iliac arteries, and is seen but rarely in the carotid arteries, bearing no constant relationship to the presence or absence of raised arterial plaques. It has been seen in subjects as young as 9 years of age, and in arteries without any evidence of intimal disease. In the vast majority of raised plaques, calcification of the internal elastic lamina is absent.

(iv) Cellular Lesions (Granulomata). These lesions are found in some $20 \%$ of raised plaques in the coronary arteries, aorta, carotid and iliac arteries. They occur in the depths of the plaque, sometimes extending through the media to the adventitial coat, and their size and structure varies considerably. Cholesterol clefts and fat deposits are common in and adjacent to such lesions and cells of many types are present, including occasional multi-nucleate giant cells of the foreign body type, macrophages containing pigment or lipid, fibroblasts, small round cells morphologically similar to lymphocytes, and occasional plasma cells (Fig. 4). Small free granules of iron-pigment are often seen in such areas. In smaller arteries and in particular the coronary arteries, with raised eccentric plaques, these granuloma-like lesions have a predilection for the angles at the junction of the plaque with the adjoining more normal segment of artery.

(v) Medial Thinning. This is a constant feature of the elevated plaque and in some instances the media is almost non-existent (Figs. 3 and 5). This

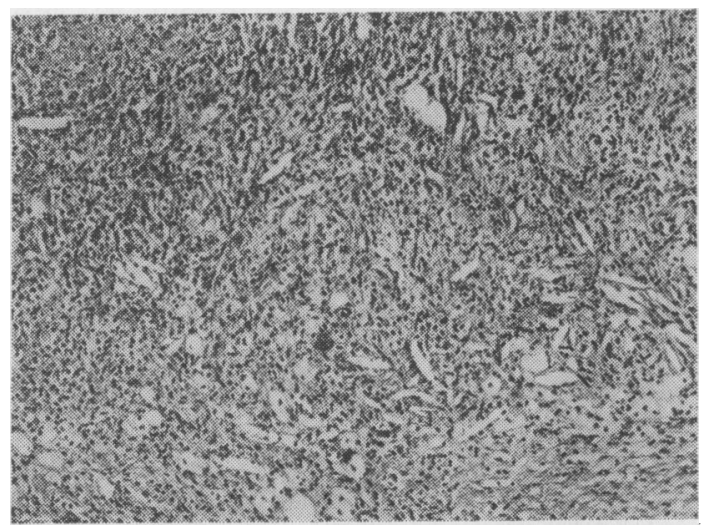

FIg. 4.-An area from a raised plaque in the carotid sinus showing a 'granuloma' with considerable cellularity, acicular cholesterol clefts and occasional giant cells of the foreign body type. Masson's trichrome $\times 100$. change is not found in association with the flaff sudanophilic plaques. Not uncommonly in severe medial thinning, the medial elastic fibres ar packed closely together, suggesting that there has been a loss of other medial components.

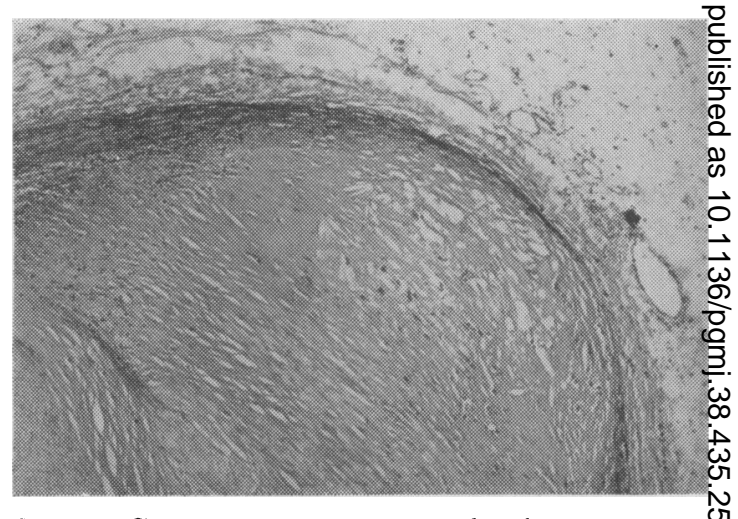

FIG. 5.-Coronary artery segment showing an extrem\& degree of medial thinning associated with a raisec plaque. The lumen of the artery is at the bottom left and contains injection mass. Masson's tric్ chrome $\times 60$.

(vi) Hamorrhage in the Plaque. Lakge accumulations of red cells in a raised plaque क्षे occasionally seen (Fig. 3) but in our survey have not found any instance of coronary occlus produced by this mechanism alone. More coms monly areas of iron pigment are found in oo adjacent to areas of cholesterol-clefted lipiळ̋ deposits. This may be evidence of a previous hæmorrhage, but similar areas are commonly seen $\vec{B}$ in organizing thrombus.

(vii) 'Fibrin' Deposits. Areas of amorphous eosinophilic material are commonly seen on the surface of raised plaques and as discrete areaso sometimes with a distinctly laminar orientation. within the dense fibrous stroma (Fig. 6). This eosinophilic material has a characteristic brickred staining behaviour with Masson's trichromê and is nearly always PTAH positive, suggesting that it may be fibrin. It stains orthochromaticalls with Azure-A and is sometimes PAS positive.

(viii) Fatty Deposits. Fat is found in everye. elevated plaque to a greater or lesser degree. IN may be present in the loose fibrous stroma on the surface of the lesions as small globules, some of which are extracellular, while others are contained within the macrophages. More commonly, and aई a characteristic of the raised plaque, confluene masses of fatty material are found in the depths ot plaque, encased in dense fibrous tissue (Fig. 3 )? These fatty areas, which correspond with the pultaceous material seen macroscopically, fre quently show numerous acicular clefts (so-calle霖 


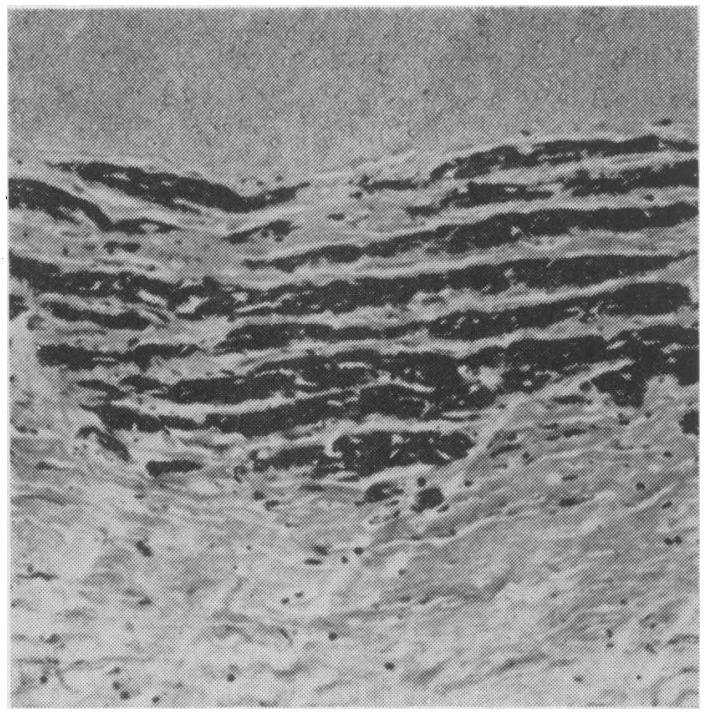

FIG. 6.- Raised plaque, from coronary artery showing areas of laminated PTAH-positive material-' fibrin'. Injection mass is visible in the lumen, at the top of the picture. PTAH $\times 20$.

cholesterol clefts) (Fig. 7) and often contain free erythrocytes or flecks of iron pigment. Within these fatty areas a finely granular eosinophilic material is often seen which may represent degenerating erythrocyte masses, but its origin remains uncertain.

(ix) Changes in the Adventitia. The adventitial changes associated with raised arterial plaques are threefold, namely an increase in fibrous tissue, an increase in vascularity, and finally a high prevalence of cellular infiltration (Fig. 8), some $75 \%$ to $80 \%$ of arterial blocks with a severe grade of disease showing cellular aggregates consisting of

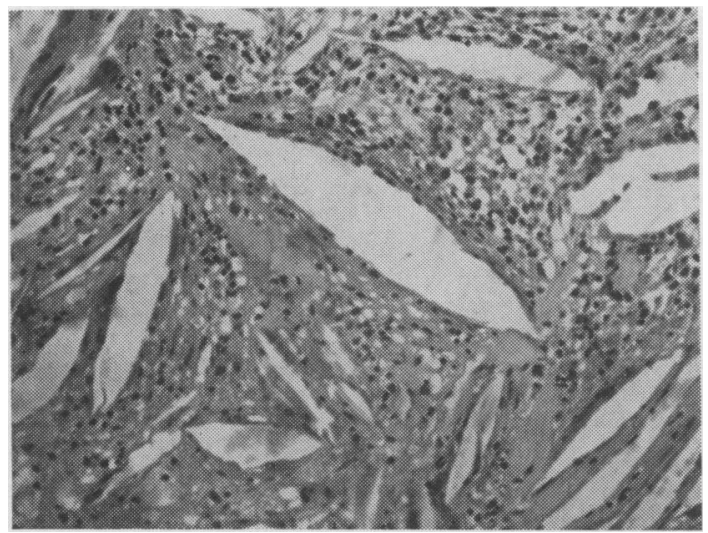

FIG. 7:- Segment of raised coronary plaque showing acicular cholesterol clefts, cellularity and amorphous hyaline material. H. \& E. $\times 200$. small lymphocytes and occasional plasma cells (Table I).

(x) Miscellaneous Changes. Changes which occur in association with raised plaques and not described above, include increased plaque vascularity, smooth muscle formation in the plaque, surface thrombosis, ulceration, and abnormalities in mucopolysaccharide staining.

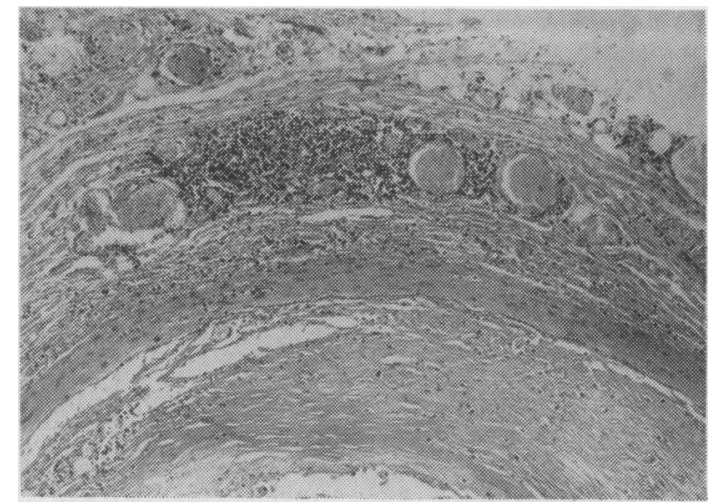

FIG. 8.-Coronary artery with a raised plaque showing adventitial lymphocytic infiltration, prominent adventitial fibrosis and increased vascularity. H. \& E. $\times 60$.

TABLE I

\begin{tabular}{|c|c|c|c|}
\hline \multirow{3}{*}{ Artery } & \multicolumn{3}{|c|}{$\begin{array}{l}\text { Prevalence of Adventitial Cellular } \\
\text { Infiltration in Arteries with Plaques } \\
\text { of a Severe Degree ( } \%)\end{array}$} \\
\hline & \multicolumn{3}{|c|}{ Cellularity Grade } \\
\hline & Absent & Slight & Marked \\
\hline Aorta & 20 & 57 & 23 \\
\hline Cervical & 22 & 67 & I I \\
\hline Iliac & 2 I & 55 & 24 \\
\hline Coronary & 25 & 52 & 23 \\
\hline
\end{tabular}

TABLE I.-The percentage prevalence of the different grades of adventitial cellular infiltration in various arteries with plaques of a severe degree derived from an unselected necropsy sample.

\section{The Possible Relationship between Flat and Raised Plaques}

It is obvious from the above descriptions that the fatty streak and the raised arterial plaque show little similarity. Indeed the only feature common to both types of lesion is the presence of fat, and although this is the usual reason for regarding them as related lesions, we consider that there is little justification for this view. From the histological point of view it is worth emphasizing that 
the lipid deposits occur characteristically in the depths of the raised arterial plaque, separated from the lumen by a dense mass of fibrous tissue, while in the flat sudanophilic plaque they are quite superficial, lying within the intima. There is one more morphological difference which cannot be orer-emphasized. Fatty streaks clearly affect the intima alone, while in raised plaques all three arterial coats are involved. This basic difference is of considerable importance and interest when one considers how 'atherosclerosis' is defined in the standard pathological texts. Thus Hall (1957) states that the lesions are due to 'intimal fibrosis and lipoidosis'; Muir (I95I) holds that the disease is "primarily a patchy lesion of the intima', and this view is shared by Boyd (r947).

Such definitions stress two points: firstly, that the disease is intimal, and secondly that it is essentially a fatty change. In other words, the disease is defined according to the histological characteristics of fatty streaking alone and has little relevance to raised arterial plaques.

These definitions are explicable if one realizes that many workers consider that fatty streaks are an early stage in the development of raised plaques. Thus Holman, McGill, Strong and Geer (1958) suggested that flat sudanophilic lesions in the aorta are a foundation for the subsequent development of raised plaques and that fatty streaks are replaced by raised plaques. However, they studied different racial groups and noted that whereas negroes had more of their aortic surface affected by fatty streaks, the subsequent development of raised lesions was significantly less in this group than in Europeans, who initially had fewer fatty streaks. Comparing the aortic lesions of South African Bantus, New Orleans negroes and New Orleans whites, Strong, Wainwright and McGill (1959) showed that, although these groups had similar amounts of aortic fatty streaking, the Bantus were much less prone to develop raised lesions. This dissociation between the amount of fatty streaking and the subsequent development of raised plaques is of considerable significance and suggests that the flat lesion does not necessarily evolve into a raised plaque and that the raised lesions may arise independently. Even if the lesions are related, it seems likely that the factors responsible for the initiation of the fatty streak differ from those responsible for its conversion into a raised plaque.

The distribution of flat and raised lesions in the aorta shows some noteworthy differences (Schwartz and Mitchell, 1961). Thus, although the upper thoracic aorta is commonly the site of confluent areas of fatty streaking, it remains relatively free from raised lesions, in contrast to the abdominal segment, which is markedly affected, although this segment shows no more fatty streaking than is found in the thoracic segment. Another dis crepancy in the distribution of these two types 7 plaque is to be found at the mouths of the intep costal arteries, where encircling raised lesions commonly occur, despite the fact that these sit $\overrightarrow{\mathrm{c}^{3}}$ are usually spared from involvement by fla th sudanophilic lesions. Another difference in dis్ㅗㅇ tribution in the aorta is that fatty streaking commonly restricted to an area along the line the intercostal arteries (Fig. I), in the midline posteriorly, with a decided tendency to spare the lateral and anterior walls of the thoracic aort whereas it is in these areas of the aortic wall that considerable numbers of raised plaques develogs with increasing age.

When such structural, topographic and ethnte differences between flat and raised plaques are com: sidered, the evidence provides little justification fơ considering them as part of one disease process Some early workers considered that they wets separate processes (Sanders, I9I I ; Ribbert, I9I 89 , while Klotz and Manning (I9II) believed that transitional lesions could be seen. Evidençe obtained from different lesions, from differeat patients of different ages, however, cannot tre accepted as establishing beyond doubt that such transition does occur, even if acceptable types of 'intermediate' lesions had been demonstrate We endorse the views of Ophüls (1933), wheे $y$ discussing the significance of fatty streaking in development of ' atheroma' remarked, ' $T$ ) microscopic appearance of the fatty streak sugges a semi-physiological deposition of lipoid material the intima ... while the atheromatous lesions from the beginning are distinctly pathological in bein accompanied by the development of new fibro丞 tissue and areas of necrosis '.

\section{The Pathogenesis of the Fatty Streak}

The histological characteristics of this type off lesion have been described, and it has been en? phasized that fatty streaking consists primarily of fatty change in the intima without any characte? istic abnormalities of the medial and adventitial coats. Whether these lesions are pathological or physiological remains uncertain, but whatever their mode of formation, fatty streaking in humân arteries shows a definite topographical localizatiof some areas being consistently prone to involvis ment and other areas spared. Such a localization suggests that hæmodynamic stresses, in particulât shearing stress, are of importance in determining where such lesions develop (Schwartz aftit Mitchell, r96r).

Recently Woolf and Crawford (1960), using fluorescein-coupled anti-human-fibrin, have consistently demonstrated the presence of fibrin aortic fatty streaks. The significance of the 
findings is uncertain. Before one can readily accept that fibrin plays any role in the pathogenesis of fatty streaks we must know to what extent a non-fatty ' normal' intima shows the presence of fibrin with this technique, as it would occasion no surprise to find fibrin in any segment of the vascular tree, diseased or normal, which is constantly in contact with fibrinogen or fibrin. Florey (1960) has emphasized the need for caution in interpreting such results, suggesting that fibrin deposits may reflect a post-mortem artefact.

It is probable that the lipids of fatty streaks are qualitatively similar to those of the circulating blood (Böttcher, Woodford, Romeny-Wachter, Boelsma-van Houte and van Gent, 1960) and the lipid may enter the arterial intima from the blood. How this process of transfer across the vascular endothelium occurs in man is not at all clear, but electron-microscopic studies may help to provide an answer. Wilens (195I) has demonstrated that the accumulation of lipids in excised human iliac arteries is dependent upon the perfusion pressure, suggesting that a process of 'filtration' might account for the development of fatty streaking.

\section{The Pathogenesis of the Raised Plaque}

There are many facets of the histopathology of raised plaques which may be of some significance in their ætiology or pathogenesis:

(a) Medial Thinning. This was recognized by Richard Thoma in 1883 , who thought that medial changes played a primary role in the development of lesions, the weakening of media resulting in focal areas of aneurysmal dilatation, which subsequently became filled in by a fibrotic repair process, thus restoring the lumen to its original size. Such a view has received support from Bork (I926) and Crawford and Levene (I953), and from our own experience we confirm that medial thinning is a characteristic finding in such plaques. Morgan (1956) suggested that the thinning might be the result of the plaque and that it might be regarded as evidence of muscular atrophy due to the splinting of the arterial wall by the plaques, and therefore akin to disuse atrophy. Whatever the significance of medial thinning in either the ætiology or pathogenesis of raised plaques, it should not be overlooked in any definition of the raised plaque which purports to be based upon the histological features of the lesions.

(b) Changes in the Adventitia. The triad of adventitial changes which occur in association with raised arterial plaques have either not been recognized or have been the subject of passing reference only. The changes include adventitial fibrosis, increased adventitial vascularity and cellular infiltration. (Schwartz and
Mitchell, 196r.) This latter change is of particular interest and requires further discussion. In Table I the percentage prevalence of adventitial cellular infiltration in arteries from different sites with a severe grade of disease is shown. It can be seen that a marked grade of cellularity occurs in some $20 \%$ of blocks examined, while slight and marked grades of cellularity combined occur in approximately $75 \%$ or more of blocks.

The infiltration shows a remarkable homogeneity, comprising small round-cells morphologically identical with lymphocytes, and an occasional plasma cell. Such cellular aggregates were noted as early as 1915 by Allbutt and they have since received mention by Ophüls (1933), Horn and Finkelstein (1940) and Nelson (1941), and more recently by Gerlis (1956) and Morgan (1956). Their presence might be interpreted as evidence of a chronic non-specific inflammatory process, but our concept of chronic non-specific inflammation is ill-defined. In some conditions thus designated and associated with abnormal lymphocytic infiltration, notably Hashimoto's thyroiditis, an auto-immune process has been implicated. We should not rule out the possibility that a similar process might play a part in the pathogenesis of the raised plaque and immunological studies on artery wall must be undertaken to evaluate this. Adventitial changes occur only in association with the raised arterial plaques and not with fatty streaks. Like the medial changes, they should not be discounted in defining the histological characteristics of the raised arterial plaque.

(c) Lipid Deposits. The presence of lipid in the raised arterial plaques is often considered as evidence that it plays a primary role in the ætiology of these lesions. There are, however, many pathological situations where fat and cholesterol deposition are encountered, including tuberculomata, necrotic tumours, etc., which no-one has ever attempted to define as disorders of lipid metabolism. The lipid of the raised arterial plaque could therefore be the end result of, and not the cause of the lesions. Much of the evidence which purports to support the hypothesis that abnormal lipid metabolism is a primary causal factor in the development of raised plaques is circumstantial. There is, for example, no doubt that a group of patients with myocardial infarction show both elevated and qualitatively abnormal serum lipid levels when compared with patients having no clinical evidence of disease (Gofman, 1959). It is common to interpret such evidence as reflecting the causal significance of abnormal lipid metabolism in 'atherosclerosis' when in fact the important difference between patients with and without myocardial infarction is the presence or 
absence of occluding coronary thrombosis (Mitchell and Schwartz, 1961).

If there is a relationship between thrombosis and abnormal lipid metabolism, as suggested by the data available, this mechanism is unknown. It is possible, however, that through the intermediary of thrombosis lipids might eventually be found to be related to the development of the raised arterial plaques, if the latter arise by a process of mural thrombosis, organization and endothelialization. This aspect will be discussed further in the next section.

As an alternative origin for the fatty material of the raised plaque, Winternitz, Thomas and Le Compte (1938) suggested that this material might result from repeated intra-mural hæmorrhage, a change which was first brought into prominence in 1936 by Patterson. This interesting mechanism might well be of some importance in substantially adding to the fatty debris seen in the depths of the raised arterial plaques. It is, however, most frequently seen in large established plaques rather than small lesions and is probably therefore a sequel to rather than the cause of these lesions.

(d) Mural Thrombosis. Thrombi in various stages of organization can frequently be seen in association with raised plaques, ranging from a laminated cellular structure characteristic of the recent lesion (Fig. 9) to the eosinophilic, often laminated areas of amorphous material which stain brick-red with Masson's trichrome and give a PTAH positive reaction for fibrin. Such changes are consistent with the findings of many other workers and in our opinion support the hypothesis originated by von Rokitansky ( 1852$)$ and emphasized by Duguid (1946, 1948, 1949) that 'atheromatous' plaques arise by a process of mural thrombosis, followed by organisation and incorporation into the arterial wall (Fig. Io). Many

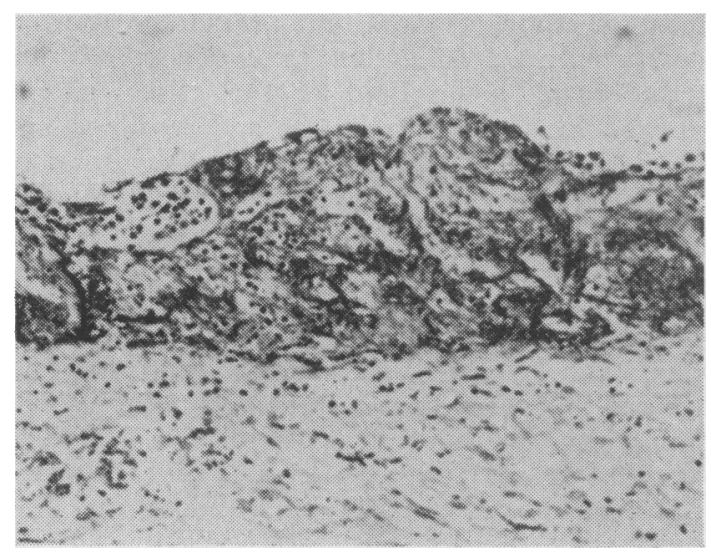

FIG. 9.-Raised aortic plaque with recent mural thrombus. PTAH $\times 140$. separate histological features of the raised plaqu can be seen during the organization of a muraf arterial thrombosis, including fibrosis, elastosi vascularization, iron pigment deposition and focat increases in metachromatic staining. Thrombi of the endocardium show some of these changes during organization and may well represent the cardiac counterpart of the raised arterial plaque (Mitchell and Schwartz, I96I.) Of all the change occurring with organization, fibrosis associatea

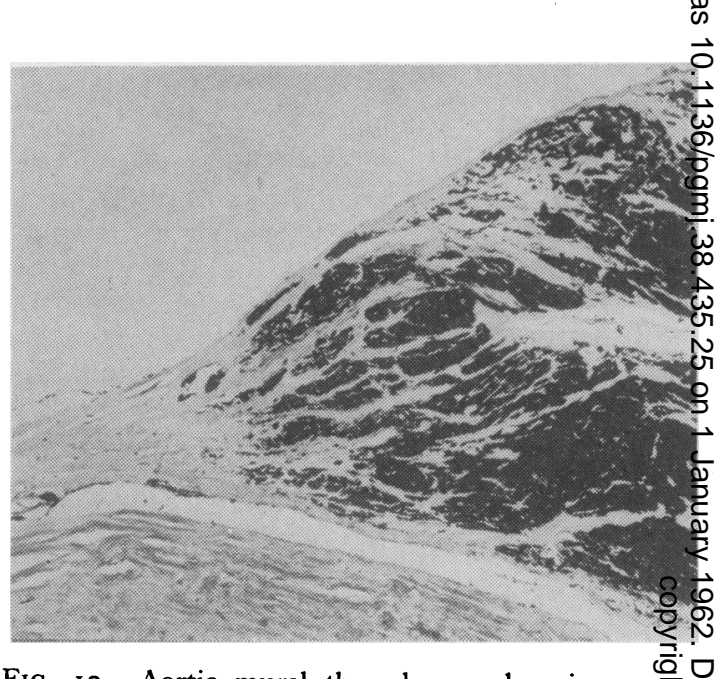

FIG. Io.-Aortic mural thrombus undergoing orga ization and completely endothelialized. The läge dark areas are PTAH-positive material (" fibrin 'o PTAH $\times 90$.

with the formation of new elastic-staining fibres $\frac{2}{78}$ the most prominent, and this material may weB constitute the dense fibro-elastic cap of the raise plaque, and also account for the laminar structure the appearance of which is consistent with periodic surface accretion. Mural thrombosis with. organization and endothelialization might occu upon areas of fatty streaking, thus providing one source of the liquid material in the depths of the raised plaque; plaques with minimal fatty changes may be the result of thrombi occurring upon non fatty areas. Intramural hæmorrhage from the new vessels of an organizing thrombus could increase. the amount of lipid in the plaque. Such vasculary ization was noted by Duguid (1946, 1948) and these observations have been confirmed by others including Dible (1958).

The mural thrombosis hypothesis of Rokitansk and Duguid is also consistent with the charactee istic patterns of localization shown by the raised plaques, for stenosing plaques occur particularly in those sites where turbulent blood flow is mose likely to develop, and non-laminar flow miglif conceivably facilitate the process of "thrombosis? 
by assisting in the accretion of platelets and other particulate material (Schwartz and Mitchell, I96r).

\section{Summary and Conclusion}

On a microscopic basis two types of arterial plaque can be distinguished: the flat sudanophilic plaque (fatty streak) and the raised plaque. While the former can be defined as a fatty lesion of the intima, the raised plaque is characterized by involvement of all three arterial coats.

Flat and raised lesions are possibly the result of unrelated processes and such a view receives support from the histological, ethnic and topographic differences between these two types of lesion. There is no clear evidence for the widely held assumption that the fatty streak is an early stage in the pathogenesis of the raised plaque.

The pathogenesis of the flat and raised plaques has been discussed. Most of the histological facets of the raised plaque are consistent with the view that they arise by the organization and endothelialization of mural thrombi. Some changes, however, are not readily accounted for on any hypothesis and are probably secondary, occurring during the evolution of the plaque, but unrelated to the primary causal mechanism. These include the medial and the adventitial changes, which may play an important role in the pathogenesis of these lesions, even though they may be of no ætiological significance.

We consider that the terms in current use, namely, atheroma, arteriosclerosis and atherosclerosis, have no concise pathological meaning and tend to imply a mechanism of causation as well as to prejudge the inter-relationship of different types of lesions. For these reasons we propose that the arterial plaques should be classified according to the macroscopic and microscopic morphology, the simplest categories on this basis being the flat sudanophilic plaque or fatty streak and the raised arterial plaque. This distinction makes no unwarranted assumptions and may provide a useful basis upon which future work can be based.

The work on which this Review is based was done while one of us, C.J.S., was the C. J. Martin Research Fellow of the National Health and Medical Research Council of Australia, and J.R.A.M. was Clinical Reseatch Fellow of the Medical Research Council.

\section{REFERENCES}

Allbutt, C. (1915): Diseases of the Arteries Including Angina Pectoris, Vol I, p. 468. London.

Aschoff, L. (1933): Arteriosclerosis, ed. E. V. Cowdry. New York: MacMillan.

Bork, K. (1926): Uber Kranzadersclerose, Virchows Arch. path. Anat., 262, 646.

Böttcher, C. J. F., Woodford, F. P., Romeny-Wachter, C. C. T. H., Boelsma-van Houte, E., and Van Gent, C. M. (1960): Fatty-Acid Distribution in Lipids of the Aortic Wall, Lancet, $1,1378$.

Boyd, W. (1947): A Text-book of Pathology, 5th edition, London, p. 400.

Crawford, T., and Levene, C. I. (1953): Medial Thinning in Atheroma, f. Path. Bact., 66, i 9.

Dible, J. H. (1958): Organization and Canalization in Arterial Thrombosis, Ibid., 75, $\mathbf{~}$.

Duguid, J. B. (1946): Thrombosis as a Factor in the Pathogenesis of Coronary Atherosclerosis, Ibid., 58, 207.

(1948): Thrombosis as a Factor in the Pathogenesis of Aortic Atherosclerosis, Ibid., 60, 57.

(1949): Pathogenesis of Atherosclerosis, Lancet, 2, 925.

Florey, H. W. (1960): Coronary Artery Disease, Brit. med. F., 2, I 329.

Gerlis, L. M. (1956): The Significance of Adventitial Cellular Infiltrations in Coronary Atherosclerosis, Brit. Heart F., $18,166$.

Gofman, J. W. (1959): Coronary Heart Disease. Oxford: Blackwell.

Hall, E. M. (1957): In Anderson's Pathology, 3rd edition. Edited W. A. D. Anderson, p. 505. St. Louis.

voN Haller, A. (1755): Cited by Long, E. R., in Arteriosclerosis, edited E. V. Cowdry, p. 26. New York: MacMillan, 1933.

Holman, R. L., McGill, H. C., Strong, J. P., and Geer, J. C. (1958): The Natural History of Atherosclerosis: The Early Aortic Lesions as Seen in New Orleans in the Middle of the 2oth Century, Amer. F. Path., 34, 209.

Horn, H., and Finkelstein, L. E. (1940): Arteriosclerosis of the Coronary Arteries and the Mechanism of their Occlusion, Amer. Heart Y., r9, 655 .

Klotz, O. (1906): A Discussion on the Classification and Experimental Production of Arterioslcerosis, Brit. med. ., 2, 1767.

-, and Manning, M. F. (r9 I I): Fatty Streaks in the Intima of Arteries, f. Path Bast., r6, 2 I 1.

Lobstein, J-G-C-F-M-(1833): Traites d'Anat. Path. Paris: Levrault.

MaRchand, F. (1904): Über Arteriosklerose, Verh. dtsch. Kongr. inn. Med., $21,23$.

Mitchell, J. R. A., and Schwartz, C. J.: The Relationship Between Myocardial Lesions and Coronary Artery Disease (awaiting publication).

MönCKEBERG, J. G. (1903): Über die reine Mediaverkalkung der Extremitz̈tenarterien und ihr Verhalten zur Arteriosklerose, Virchows Arch. path. Anat., r71, 141.

Morgan, A. D. (1956): The Pathogenesis of Coronary Occlusion, p. 60, 64. Oxford: Blackwell.

Muir, R. (195I): Textbook of Pathology, 6th edition, edited D. F. Cappell, p. $288 . \quad$ London.

Nelson, M. G. (I94I): Intimal Artery Hæmorrhage as a Factor in the Causation of Coronary Occlusion, F. Path bact., 53, 105 .

Opнüis, W. (1933): Arteriosclerosis, edited E. V. Cowdry, p. 254. New York: MacMillan.

ParE, A. (1 575): Cited by Long, E. R., in Arteriosclerosis, edited E. V. Cowdry, p. 28. 1933. New York: MacMillan.

Patterson, J. C. (1936): Vascularization and Hæmorrhage of the Intima of Arteriosclerotic Coronary Arteries, Arch. Path., 22, 313 . 
Rabson, S. M. (1954): Letter to the Editor, Amer. F. clin. Path., 24, 472.

RibBert, H. (I918), Die Arteriosklerose, Dtsch. med. Wschr., 44, 953.

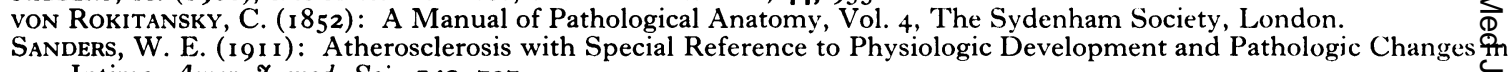
Intima, Amer. F. med. Sci., 142, 727.

Schwartz, C. J., and Mitchell, J. R.A.: Observations on the Localization of Arterial Plaques (awaiting publication.

- - - Cellular Infiltration of the Human Arterial Wall Associated with Atheromatous Plaques (awaitifing publication).

Strong, J. P., Wainwright, J., and McGill, H. C. (1959): Atherosclerosis in the Bantu, Circulation, 20 , ir 8.

Tнома, R. (1883): Uber die Abhängigkeit der Bindegewebsneubildung in der Arterienintima von den Mechanisch Bedingungen des Blutumlaufes. I. Die Rückwirkung des Verschlusses der Nabelarterien und des arteriös Ganges auf die Structur der Aortenwand, Virchows Arch. path. Anat., 93, 443.

Wilens, S. L. (1951): The Experimental Production of Lipid Deposition in Excised Arteries, Science, 114, 389.

Winternitz, M. C., Thomas, R. M., and LeCompte, P. M. (1938): The Biology of Arteriosclerosis. Baltimore: Thomas.

Woolf, N., and Crawford, T. (1960): Fatty Streaks in the Aortic Intima Studied by an Immuno-Histo-chemieal Technique, F. Path. Bact., 80, 405 .

World Health Organization (1958): Report of a Study Group, Wld. Hlth. Org. techn. Rep. Ser., i 43. 